\title{
(9) A Pathological Study
}

\author{
Syoichi OTSU \\ Department of Pathology, Faculty of Medicine, University of Tokyo
}

The "intermediate coronary syndrome" (Vakil), a "variant form of angina pectoris" (Prinzmetal et al.) etc. are all clinically established concepts. It may be, therefore, desired to be disputed, whether these conceps are able to be defined morphologically or not. Accordingly, the author and his co-workers, on the one hand, collected the cases having ischemic myocardial lesions, whether minute scars or large infarcts are concerned, and studied them both clinically and morphologically. They also, on the other hand, examined the autopsies which clinically had ever shown one or repeated cardiac attack which may be corresponding to "intermediate coronary syndrome".

Material and Methods:

1. Routine 342 adult autopsies (217 male and 125 female) at the Branch Hospital of the University of Tokyo during past 5 years (1962-1966) and 106 autopsies (29 male and 77 female) at Yokuhukai Geriatric Hospital during past 13 months (from Jan. 1965 to Jan. 1966) were studied. Thirty-four cases (19 male and 15 female) among the former group and 48 (14 male and 34 female) among the latter came under autopsies with any ischemic myocardial change.

2. Then, among 124 autopsies (69 male and 55 female) originated from 4th Department of Internal Medicine (Director: Prof. Dr. T. Kobayashi) during past 5 years, were selected 2 cases who had ever suffered from cardiac attack resembling "intermediate form", while 15 among 266 (86 male and 180 female) during past 30 months at Yokuhukai Geriatric Hospital in the same manner.

All cases concerned were examined macroscopically as well as histologically, especially regarding their myocardial changes, coronary sclerosis and thrombosis.

Results :

1. Cases with ischemic myocardial changes

According to size and number of ischemic myocardial lesions, 82 cases selected were divided into four groups as follows.

Group Ia-Cases with one or a few minute myocardial scars- 24 cases

Group Ib-Cases with scattered minute and/or small myocardial scars-24 cases

Group II-Cases with one or a few small myocardial infarcts-13 cases

Group III-Cases with one or more large myocardial infarcts-21 cases

Number of cases with some electrocardiographic abnormalities among group Ia, Ib, II and III is $16,19,10$ and 20 , respectively, including as a whole 4 having shown 
Table 1. Clinical Data of Cases Diagnosed as "Intermediate Coronary Syndrome"

\begin{tabular}{|c|c|c|c|c|c|c|c|c|c|c|c|}
\hline \multirow{2}{*}{$\begin{array}{c}\text { Autopsy } \\
\text { No. }\end{array}$} & \multirow{2}{*}{ Age } & \multirow{2}{*}{ Sex } & \multirow{2}{*}{ Main diseases } & \multirow{2}{*}{$\begin{array}{l}\text { Hyper- } \\
\text { tension }\end{array}$} & \multicolumn{2}{|c|}{$\begin{array}{c}\text { Cardiac } \\
\text { attack }\end{array}$} & \multirow{2}{*}{ GOT } & \multirow{2}{*}{ GPT } & \multirow{2}{*}{$\begin{array}{l}\text { No. of } \\
\text { leuco- } \\
\text { cyte }\end{array}$} & \multicolumn{2}{|c|}{ Electrocardiogram } \\
\hline & & & & & $\begin{array}{l}\text { Freq- } \\
\text { uency }\end{array}$ & Sever- & & & & Main changes & $\begin{array}{l}\text { Dura- } \\
\text { tion }\end{array}$ \\
\hline B. 1226 & 64 & $\mathrm{~F}$ & Malnutrition & - & $(+)$ & + & 19 & 8 & 11,000 & $\begin{array}{l}\operatorname{ST} \uparrow\left(V_{1,5,6}\right) \\
\text { trans. } Q S\left(V_{2-4}\right)\end{array}$ & $4 \mathrm{~d}$. \\
\hline Y. 2875 & 91 & $\mathrm{~F}$ & $\begin{array}{l}\text { Emaciation, } \\
\text { purulent } \\
\text { cystitis }\end{array}$ & H & $\stackrel{+}{(1)}$ & $H$ & & & & $\begin{array}{l}\text { Cor. } T \text { (II, III, } \\
\left.\mathrm{aV}_{\mathrm{F}}, \mathrm{V}_{3-6}\right)\end{array}$ & $2 \mathrm{~m}$. \\
\hline Y. 2750 & 89 & $\mathrm{~F}$ & $\begin{array}{l}\text { Senile emacia- } \\
\text { tion }\end{array}$ & + & $(+)$ & \pm & 35 & 14 & 6,100 & Neg. $T\left(\mathrm{~V}_{3-6}\right)$ & $4 \mathrm{~m}$. \\
\hline Y. 2840 & 81 & $\mathrm{~F}$ & $\begin{array}{l}\text { Carcinoma of } \\
\text { caecum }\end{array}$ & H & $\stackrel{+}{(1)}$ & + & 17 & & 6,100 & $\begin{array}{l}\text { Neg. T(II, III, } \\
\text { a VF, V V } \\
S T \downarrow\left(\mathrm{V}_{5}\right)\end{array}$ & $8 \mathrm{~m}$. \\
\hline Y. 2923 & 73 & $\mathrm{~F}$ & $\begin{array}{l}\text { R. hemiparaly- } \\
\text { sis }\end{array}$ & H & $+(1)$ & \pm & & & & $\begin{array}{l}\text { Cor. } T\left(\mathrm{~V}_{2-5}\right), \\
S T \downarrow\left(\mathrm{V}_{5,6}\right)\end{array}$ & $45 \mathrm{~d}$ \\
\hline Y. 2948 & 77 & $\mathrm{~F}$ & $\begin{array}{l}\text { Pneumonia, } \\
\text { tbc., cardiac } \\
\text { insufficiency }\end{array}$ & - & $\stackrel{+}{(1)}$ & \pm & & & & Cor. $T\left(\mathrm{~V}_{\mathrm{2}-4}\right)$ & $9 \mathrm{~d}$. \\
\hline Y. 2720 & 70 & $\mathrm{~F}$ & $\begin{array}{l}\text { Acute fatal } \\
\text { hepatitis }\end{array}$ & H & $\stackrel{+}{(1)}$ & \pm & & & & $\begin{array}{l}\text { Cor. } T\left(\mathrm{aV}_{\mathrm{L}}\right. \\
\left.\mathrm{V}_{4-6}\right), S T \downarrow\left(\mathrm{V}_{5}\right)\end{array}$ & $38 \mathrm{~d}$. \\
\hline Y. 2952 & 76 & $\mathrm{M}$ & $\begin{array}{l}\text { Cerebellar } \\
\text { hemorrhage }\end{array}$ & H & $\stackrel{+}{(1)}$ & \pm & & & & $\begin{array}{l}\text { Cor. } T\left(\mathrm{a}_{\mathrm{a}} \mathrm{V}_{\mathrm{L}}\right), \\
S T \downarrow\left(\mathrm{V}_{5}\right)\end{array}$ & $5 \mathrm{~m}$. \\
\hline Y. 2946 & 74 & $\mathrm{~F}$ & $\begin{array}{l}\text { Pulmonary } \\
\text { emphysema, } \\
\text { enteritis }\end{array}$ & - & $+\frac{+}{4}$ & H & $\begin{array}{l}48 \\
25\end{array}$ & $\begin{array}{l}13 \\
11\end{array}$ & $\begin{array}{r}10,200 \\
6,250\end{array}$ & $\begin{array}{l}\text { Giant Cor. } T \\
\left(\mathrm{~V}_{3-5}\right), 4 \text { times }\end{array}$ & $\begin{array}{r}3 \mathrm{~d} . \\
\sim 5 \mathrm{~m} .\end{array}$ \\
\hline Y. 2926 & 69 & $\mathrm{~F}$ & $\begin{array}{l}\text { CVI, myocar- } \\
\text { dial damage }\end{array}$ & + & $\stackrel{+}{(1)}$ & \pm & 33 & 14 & & $\begin{array}{l}\text { Cor. } T\left(\mathrm{~V}_{2-5}\right), \\
S T \downarrow\left(\mathrm{V}_{4-6}\right)\end{array}$ & $7.5 \mathrm{~m}$. \\
\hline Y. 2734 & 91 & $\mathrm{~F}$ & $\begin{array}{l}\text { Cerebral } \\
\text { infarction, } \\
\text { pulmonary } \\
\text { thrombosis }\end{array}$ & H & $\stackrel{+}{(1)}$ & + & 30 & 10 & 5,300 & $\begin{array}{l}\text { Giant Cor. } T(\mathrm{I}, \\
\left.\mathrm{II}, \mathrm{V}_{\mathrm{L}}, \mathrm{V}_{2-5}\right), \\
\operatorname{ST} \downarrow\left(\mathrm{V}_{4-6}\right)\end{array}$ & $5 \mathrm{~m}$. \\
\hline Y. 2737 & 79 & $\mathrm{M}$ & $\begin{array}{l}\text { R. hemiparesis, } \\
\text { gastric cancer }\end{array}$ & - & $\stackrel{H}{(1)}$ & $H$ & 20 & 2 & 4,450 & Neg. T( $\left(\mathrm{V}_{4-6}\right)$ & $1 \mathrm{~m}$. \\
\hline Y. 2849 & 76 & $\mathrm{~F}$ & $\begin{array}{l}\text { Cerebral } \\
\text { hemorrhage, } \\
\text { pneumonia }\end{array}$ & + & $\stackrel{\#}{(1)}$ & + & & & & $\begin{array}{l}\text { Giant Cor. } T \\
\left(\mathrm{~V}_{3-6}\right)\end{array}$ & $22 \mathrm{~m}$. \\
\hline B. 987 & 72 & $\mathrm{M}$ & $\begin{array}{l}\text { Myoc } \\
\text { infa }\end{array}$ & + & $\stackrel{H}{H}$ & $H$ & 19 & & 8,400 & $\begin{array}{l}S T \downarrow\left(\mathrm{I}, \mathrm{II}, \mathrm{V}_{4}\right. \\
6), N e g . T(\mathrm{III}, \\
\left.{ }_{\mathrm{a}} \mathrm{V}_{\mathrm{L}}, \mathrm{aV}_{\mathrm{F}}, \mathrm{V}_{3}\right)\end{array}$ & $15^{\prime}$ \\
\hline Y. 2745 & 78 & $\mathrm{~F}$ & $\begin{array}{l}\text { Myocardial in- } \\
\text { farction, rup- } \\
\text { ture of aortic } \\
\text { aneurysm }\end{array}$ & + & $\stackrel{+}{(1)}$ & $+\sim H$ & & & & $\begin{array}{l}\text { Cor } T \text { and } \\
S T \downarrow \text { (II, III, } \\
\left.{ }_{\mathrm{a}} \mathrm{V}_{\mathrm{F}}\right)\end{array}$ & $5 \mathrm{~m}$. \\
\hline Y. 2842 & 79 & $\mathrm{~F}$ & $\begin{array}{c}\text { Myocardial } \\
\text { infarction }\end{array}$ & - & $\stackrel{H}{(1)}$ & $+\sim H$ & & & & $\begin{array}{l}\text { Cor. } T\left(V_{2-4}\right), \\
S T \downarrow\left(V_{3-5}\right)\end{array}$ & $27 \mathrm{~m}$. \\
\hline Y. 2735 & 75 & F & $\begin{array}{c}\text { Myocardial } \\
\text { infarction }\end{array}$ & H & $\begin{array}{l}\# \\
(1)\end{array}$ & $1+\sim$ & & & & $\begin{array}{l}\text { Neg. T and } \\
S T_{\downarrow}\left(\mathrm{V}_{2-6}\right)\end{array}$ & $14 \mathrm{~d}$. \\
\hline
\end{tabular}

Note. ( ) : Number of attacks diagnosed as "intermediate form" fr: frequent, d.: days, m.: months, ': minutes

Autopsy No. underlined: cases diagnosed as typical "Intermediate coronary syndrome" 
Table 2. Pathological Data of Cases Diagnosed as "Intermediate Coronary Syndrome"

\begin{tabular}{|c|c|c|c|c|c|c|c|}
\hline \multirow{2}{*}{$\begin{array}{l}\text { Autopsy } \\
\text { No. }\end{array}$} & \multirow{2}{*}{$\begin{array}{c}\text { Heart } \\
\text { weight }\end{array}$} & \multicolumn{3}{|c|}{$\begin{array}{l}\text { Coronary } \\
\text { sclerosis }\end{array}$} & \multirow{2}{*}{$\begin{array}{c}\text { Coro- } \\
\text { nary } \\
\text { throm- } \\
\text { bosis }\end{array}$} & \multirow{2}{*}{ Main myocardial changes } & \multirow{2}{*}{ Remarks } \\
\hline & & $\begin{array}{l}1 . \\
\text { des }\end{array}$ & cir & r. & & & \\
\hline B 1226 & 210 & \pm & \pm & + & - & $\begin{array}{l}\text { Diffuse chronic deger } \\
\text { muscle fiber with pa: }\end{array}$ & Myocardosis? \\
\hline Y 2875 & 190 & H & III & H & - & Diffuse fibrosis $(+)$ & Fibrosis? \\
\hline Y 2750 & 255 & + & + & + & - & $\begin{array}{l}\text { A few small scars (ant.), diffuse } \\
\text { fibrosis }\end{array}$ & Fibrosis? \\
\hline Y 2840 & 335 & + & + & H & - & $\begin{array}{l}\text { Small or large degenerative foci, } \\
\text { scattered fibrosis }(+)\end{array}$ & Fibrosis? \\
\hline Y 2923 & 300 & + & - & + & - & $\begin{array}{l}\text { A few minute scars, local. fibrosis, } \\
\text { Scattered minute necrotic foci (post.) }\end{array}$ & Minute scars? \\
\hline Y 2948 & 370 & + & + & H & - & $\begin{array}{l}\text { Multiple small scars, diffuse fibrosis } \\
\text { (ant., post., apical), scattered } \\
\text { necrotic foci }\end{array}$ & Necrotic foci? \\
\hline Y 2720 & 390 & H & H & m & - & Scattered minute scars, diff. fibrosis & Minute scars? \\
\hline Y 2952 & 350 & Ht & 世 & $H$ & $\begin{array}{l}+(1 \\
\text { circ. } \\
\text { des. })\end{array}$ & $\begin{array}{l}\text { Scattered small scars, diffuse fibrosis, } \\
\text { necrotic foci (lat., post., ant.) }\end{array}$ & Small scars? \\
\hline Y 2946 & 330 & H & H & m & - & $\begin{array}{l}\text { Diffuse fibrosis, scattered small } \\
\text { scars, a few necrotic foci }\end{array}$ & Small scars? \\
\hline Y 2926 & 230 & H & H & $H$ & $\begin{array}{l}+(\mathrm{l} . \\
\operatorname{circ} .)\end{array}$ & $\begin{array}{l}\text { A few minute scars or fibrosis, } \\
\text { scattered necrotic foci (post.) }\end{array}$ & Minute scars? \\
\hline Y 2734 & 290 & H & H & +4 & - & $\begin{array}{l}\text { Scattered mintue scars, necrotic foci, } \\
\text { diffuse fibrosis }\end{array}$ & Minute scars? \\
\hline Y 2737 & 305 & \# & - & H & - & A few small scars & Small scars? \\
\hline Y 2849 & 265 & H & + & + & - & $\begin{array}{l}\text { A few minute scars, scattered } \\
\text { necrotic foci }\end{array}$ & Minute scars? \\
\hline В 987 & 410 & H & H & HH & $+(\mathrm{r})$. & $\begin{array}{l}\text { it infarcts (post.), } \\
s \text { (ant.) }\end{array}$ & $\begin{array}{l}\text { Scars and } \\
\text { necrosis? }\end{array}$ \\
\hline Y 2745 & 240 & \# & $H$ & + & - & $\begin{array}{l}\text { Large old infarct (post.), scattered } \\
\text { necrotic foci (post., ant.), small } \\
\text { scars, diffuse fibrosis (ant.) }\end{array}$ & Small scars? \\
\hline Y 2842 & 340 & H & 世 & m & $+(\mathrm{r}:)$ & Large old infarct (lat., post., ant.) & $\begin{array}{l}\text { Preceded by } \\
\text { infarct? }\end{array}$ \\
\hline Y 2735 & 290 & $H$ & $H$ & \# & - & $\begin{array}{l}\text { Large old infarct (post.), wide } \\
\text { recent deg. or necrosis (ant.), diff. } \\
\text { fibrosis, scattered minute scars (ant.) }\end{array}$ & Minute scars? \\
\hline
\end{tabular}

Note. I. des: left anterior descending coronary artery

1. cir : left circumflex coronary artery

r. : right coronary artery

electrocardiogram of "intermediate form" during or after cardiac attacks. It was noteworthy that two of these cases proved large myocardial infarcts. Each of remainder belongs to group Ia and Ib, respectively.

2. Cases having experienced cardiac attacks resembling "intermediate coronary syndrome" 
Both main clinical and pathological data of 17 autopsies who had ever suffered from cardiac attacks resembling "intermediate form" are shown in the Table 1 and 2 , respectively. Two cases at the top of these tables proved no ischemic myocardial lesions, while 2 cases which come next showed changes corresponding to the group Ia mentioned above and following 9 to the group Ib. Four cases at the bottom of tables fell under the group III. Therefore, in 11 of 17 cases were encountered only minute or small myocardial scars and/or necrotic foci.

Four of 17 autopsies, that is Y2750, Y2946, Y 2735 and B 987, had experienced cardiac attacks diagnosed as typical "intermediate form" after examining thoroughly, and 2 of them proved large myocardial infarcts. Some cases including 3 of them are briefly described as follows.

Case 1. Y 2946. A 74-yr-old woman. Her main diseases were pulmonary emphysema and acute enteritis. She experienced four times of cardiac attacks diagnosed as typical "intermediate form". She expired one year and half after the last attack. Necropsy revealed somewhat diffuse fibrosis, scattered small scars and a few small necrotic foci in the myocardium of left ventricle. The small scars may be changes resulting from the above mentioned attacks.

Case 2. Y 2735. A 75-yr-old woman was repeatedly attacked by angina pectoris during past several years and then by a posterior myocardial infarction. The electrocardiogramm obtained at this time revealed some changes corresponding to "the intermediate form" in the anterior wall of left ventricle. Two weeks later these changes disappeared, and 14 months thereafter she died from an attack of anterior infarction. Necropsy, revealed both wide-spread old posterior and recent anterior infarcts, and several small scars which may be consequent on the intermediate coronary attack.

Case 3. B 987. A 72-yr-old man suffered from repeated intermediate coronary attacks which usually recurred within approximately 15 minutes. These attacks were pursued by the telemeter. He died from suspicious myocardial infarction. Necropsy revealed a wide-spread scarred posterior infarct and scattered many small recent as well as old necrotic foci. These small lesions may have resulted from repeated intermediate attacks, while the infarct was probably widened by repeated intermediate attacks.

Case 4. Y 2842. A 79-yr-old woman was repeatedly attacked by angina pectoris during past 5 years. Approximately 3 years before her death there was a suspicion of an antero-lateral infarction by the vectorcardiography. Necropsy revealed a widespread old infarct, the center of which was the lateral wall of left ventricle. This infarct may already be formed prior to the possible intermediate attack.

Case 5. Y 2948. A 77-yr-old woman had a possible intermediate coronary attack 20 days before her death. Necropsy revealed a few small organizing necrotic foci, which may be changes resulting from the above mentioned cardiac attack, while there were several scattered small scars and recent necrotic foci in the anterior wall of left ventricle.

There were also a few cases which showed some interesting findings. A 73-yr- 
old woman ( $\mathrm{Y}$ 2923) had a few small scars possibly corresponding to an intermediate coronary attack 4 months prior to her death, while there were also found several necrotic foci caused by the thrombosis of intramyocardial coronary branches. A 91yr-old woman (Y 2875) had no significant myocardial change except for a diffuse fibrosis of slight degree, and another 64-yr-old woman (B 1226) had only myocardosislike changes which were presumably caused by malnutrition.

Comment :

According to facts mentioned above, the myocardial changes of patients who had undergone typical or possible intermediate coronary attacks were usually similar to ones due to angina pectoris or myocardial infarction. Therefore, there are no morphological changes specific to the intermediate coronary attacks. Nevertheless, there were frequently encountered minute or small scars and/or necrotic foci in the myocardium of these patients, while a few cases had no pathologic lesions possibly correspondent to the attacks. Several cases had myocardial infarcts, all of which were old except for one of a patient (Y 2735) who died from a recent myocardial infarction. It is noticeable that in some cases myocardial infarcts were presumably formed prior to the intermediate coronary attacks. As mentioned above, however, only a minority of cases showing ischemic myocardial changes, if recent or old, went through intermediate attacks.

These facts may indicate, on one hand, that there are few patients, whose coronary attacks are observed under the condition that the attacks can be sufficiently compared with criteria of "the intermediate form" of coronary attacks, and on the other hand, that "the intermediate form" is hardly characterized by morphological findings.

Acknowledgment: The author wishes to express his gratitude to Dr. Fujio Terasawa for his help in examining autopsies at Yokuhukai Geriatric Hospital. 